\title{
A Comparative Study of the Trends of Imported Dengue Cases in Korea and Japan 2011 - 2015
}

\author{
Shinichiro Miki ${ }^{\mathrm{a}}$, Won-Chang Lee ${ }^{\mathrm{b}}$, Myeong-Jin Lee ${ }^{\mathrm{a}, \mathrm{c}}$
}

\begin{abstract}
Background: Dengue is a mosquito-borne febrile disease that represents a major public health problem in tropical and subtropical areas. Even though Korea and Japan are not the regions where dengue is epidemic, there have been many imported cases in both countries, and in increasing numbers. A better understanding of the characteristics of the prevalence of dengue and the recent trends in these neighboring countries may provide information to promote improvement and control strategies for both. The present study investigated the epidemiological status of imported dengue cases in Korea and Japan between 2011 and 2015, and compared their characteristics.
\end{abstract}

Methods: We analyzed the annual transition of prevalence, geographic origin of dengue infection, and seasonal distribution of occurrence. The raw data on dengue cases in Korea were obtained from the Korea Center for Disease Control and Prevention infectious diseases surveillance website and Korean Statistical Information Service website. Data on dengue cases in Japan were obtained from the National Institute of Infectious Diseases, Japan's Infectious Disease Surveillance Center website.

Results: There were 893 reported cases in Korea and 1,054 in Japan between 2011 and 2015. Cumulative incidence per 100,000 overseas travelers from Japan did not substantially differ from that for Korea (1.22 vs. 1.16, respectively), despite Japan's population being roughly 2.5 times larger. These results suggest Koreans engage in overseas travel more than Japanese. For Korea and Japan, Southeast Asia was the region accounting for the most cases of infection $(89.4 \%$ vs. $75.4 \%$, respectively). Notably, the Philippines and Indonesia were, respectively, the leading origin countries for Korean (38.1\%) and Japanese $(23.3 \%)$ cases. Seasonal distribution shows August and September were the months in which the largest number of cases occurred in Korea and Japan, respectively. These differences evidently derive from characteristics of travel destinations and timing of holidays.

Manuscript accepted for publication May 12, 2017

aDepartment of Health and Nutrition, Faculty of Health and Nutrition, Otemae University, Osaka 540-0008, Japan

${ }^{\mathrm{b} C o l l e g e}$ of Veterinary Medicine, Konkuk University, Seoul 05029, Korea ${ }^{\mathrm{c} C}$ Corresponding Author: Myeong-Jin Lee, Department of Health and Nutrition, Faculty of Health and Nutrition, Otemae University, 2-1-88 Otemae, Chuo-ku, Osaka 540-0008, Japan. Email: myeong@otemae.ac.jp

doi: https://doi.org/10.14740/jocmr3022w
Conclusion: Based on the recent increasing trend in imported dengue cases in both countries, a more rigorous information system that can effectively provide warning of dengue risk and means of prevention for travelers headed to at-risk areas is urgently needed in both countries.

Keywords: Dengue; Imported; Overseas travel; Korea; Japan

\section{Introduction}

Dengue, including dengue fever and dengue hemorrhagic fever, is a mosquito-borne (Aedes species) febrile disease that represents a major public health problem in tropical and subtropical areas. The disease is transmitted mainly via the bite of Aedes mosquitoes infected with the dengue virus, which belong to the genus Flavivirus of the family Flaviviridae. There are four distinct, but closely related, serotypes of the virus that cause dengue (DENV-1 to 4) [1-3]. The Aedes aegypti mosquito, the principal vector, is usually present in regions where winter isotherm is maintained at greater than $10^{\circ} \mathrm{C}$ [1]. In the last 50 years, dengue incidence has risen 30 -fold globally in line with explosive geographic expansion to new countries. Around 50 100 million infections occur annually worldwide [1, 4].

In Korea, which is located in the temperate zone of Northeast Asia, no domestic dengue cases have ever been reported. The first imported dengue case, a woman who had traveled to Sri Lanka [3, 5], was reported in 1995, after which the number of such cases grew considerably, especially from the 2000s. While Japan is a neighboring country of Korea, domestic dengue infections have occurred on a number of occasions. From 1942 to 1945, a severe dengue epidemic hit the Osaka and Kyushu regions, with over 200,000 cases $[6,7]$ reported in that period. After that, no domestic infections had been reported. However, in 2014, Tokyo reported 162 domestic cases [8].

Even though domestic outbreaks of dengue have not been confirmed in Korea, and have not been routine in Japan, there have been many imported cases in both, and in increasing numbers $[9,10]$. In light of this situation, in Korea the Infectious Disease Control and Prevention Act classified dengue among group IV notifiable infectious diseases. Japan's Infectious Disease Control Law also, since 1999, has classified dengue as a category IV notifiable infectious disease. Despite the classification, numbers of reported cases in both countries 


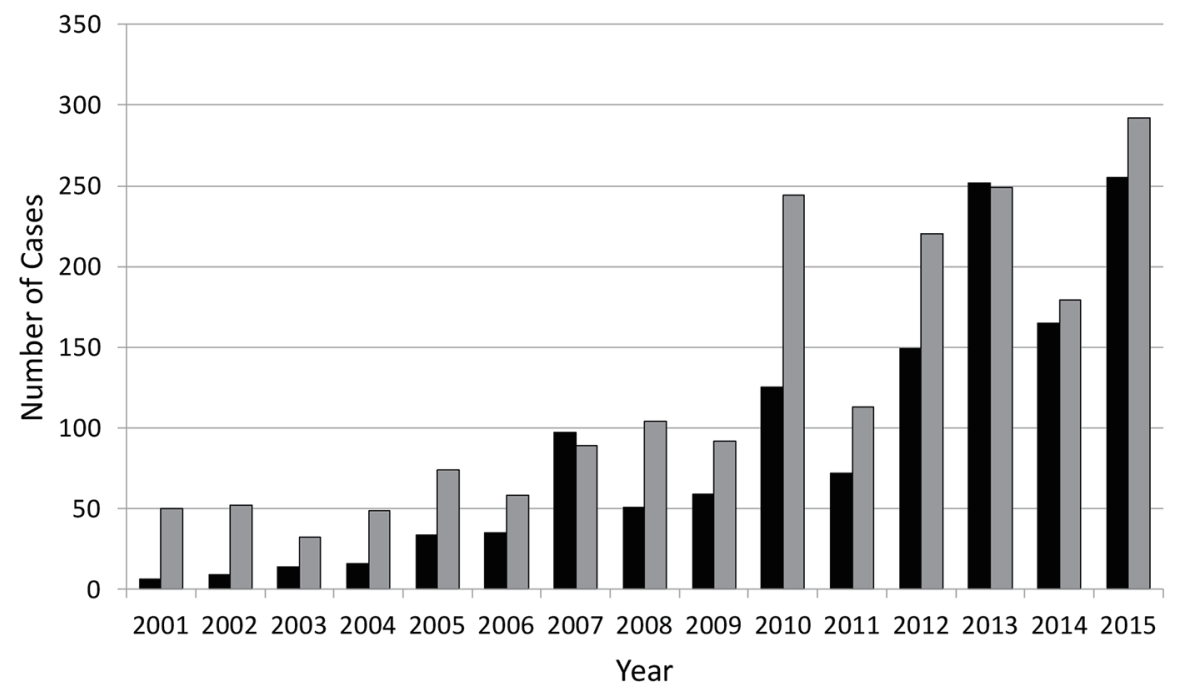

Figure 1. Annual change of dengue cases imported into Korea and Japan, 2011 - 2015. Bars represent the number of reported cases each year in Korea (black) and Japan (gray).

are rapidly increasing in recent years $[9,10]$, necessitating a system for warning about dengue infection to people planning overseas travel.

A better understanding of the characteristics of the prevalence of dengue and the recent trends in these neighboring countries may provide information to promote improvement and control strategies for both. The present study investigated the epidemiological status of imported dengue cases in Korea and Japan between 2011 and 2015, and compared their characteristics.

\section{Materials and Methods}

We analyzed the annual transition of prevalence, geographic origin of dengue infection, and seasonal distribution of occurrence. The raw data on dengue cases in Korea were obtained from the Korea Center for Disease Control and Prevention infectious diseases surveillance website [9] and Korean Statistical Information Service website [11]. Data on dengue cases in Japan were obtained from the National Institute of Infectious Diseases, Japan's Infectious Disease Surveillance Center website [10].

\section{Results and Discussion}

As shown in Figure 1, dengue cases began to sharply increase in both countries from around 2010. Especially in 2015, the highest recorded numbers of imported cases in the period since 2001 were reported: 255 for Korea and 292 for Japan. This tendency aligns with increasing worldwide outbreaks $[1,2]$.

Table 1 shows the number of total cases, cumulative incidence per 100,000 overseas travelers, and travel destinations of imported dengue cases in Korea and Japan between 2011 and 2015. A total of 893 cases in Korea and 1,054 cases in
Japan occurred during that period. Cumulative incidence in Korea was 1.16 per 100,000 overseas travelers in that time, and annual cases were 72 - 255. Cumulative incidence in Japan was 1.22 over the same period, with 113 - 299 annual cases. Total reported cases of dengue in Japan $(1,054)$ were about 1.2 times those in Korea (893), and cumulative incidence of dengue fever per 100,000 overseas travelers in Japan did not greatly differ from that in Korea (1.22 vs. 1.16, respectively). The number of annual overseas travelers of Japan is only 1.1 times higher than that of Korea during the period (861 million vs. 767 million, respectively) $[12,13]$, despite the total population of Japan being about 2.5 times that of Korea (127 million vs. 50 million as of 2015, respectively). These data suggest people from Korea engage in overseas travel more than those from Japan.

As shown in Table 1, areas suspected as sources of imported dengue cases in Korea were: Southeast Asia (89.4\% of total cases), South Asia (7.5\%), East Asia (0.6), and others or unknown (2.6\%). In Japan, these were Southeast Asia (75.4\%), South Asia (12.3\%), East Asia (0.5\%), and others or unknown $(11.8 \%)$. In both countries, Southeast Asia was the region from which the most cases originated, and the case percentage for Korea was higher than for Japan $(89.4 \%$ vs. $75.4 \%)$. Notably, the Philippines were the foremost source of Korean cases (38.1\%), while for Japan, Indonesia was highest (23.3\%). The Philippines, Indonesia, and Thailand were the three leading countries of dengue infection origin for both Korea and Japan, representing a collective $63.4 \%$ and $58.0 \%$ of total cases, respectively. The frequency of infection originating in South Asian countries was much lower for those from Korea than those from Japan (7.5\% vs. 12.3\%). These differences evidently derive from the countries' characteristics of travel destinations and timing of holidays.

In recent years, travelers from both Korea and from Japan are showing greater tendency to visit developing countries in Asia, where risk of dengue infection is substantially higher 
Table 1. Characteristics of Dengue Cases Imported Into Korea and Japan, 2011 - 2015

\begin{tabular}{|c|c|c|}
\hline & \multicolumn{2}{|c|}{ No. of cases $(\%)$} \\
\hline & Korea & Japan \\
\hline No. of total cases & 893 & 1,054 \\
\hline Cumulative incidence per 100,000 oversea travelers & 1.16 & 1.22 \\
\hline \multicolumn{3}{|l|}{ Suspected source country/areas } \\
\hline Southeast Asia & $798(89.4)$ & $795(75.4)$ \\
\hline The Philippines & $340(38.1)$ & $232(22.0)$ \\
\hline Indonesia & $103(11.5)$ & $246(23.3)$ \\
\hline Thailand & $123(13.8)$ & $134(12.7)$ \\
\hline Vietnam & $45(5.0)$ & $21(2.0)$ \\
\hline Cambodia & $66(7.4)$ & $38(3.6)$ \\
\hline Laos & $17(1.9)$ & $8(0.8)$ \\
\hline Malaysia & $52(5.8)$ & $71(6.7)$ \\
\hline Myanmar & $33(3.7)$ & $29(2.8)$ \\
\hline Singapore & $18(2.0)$ & $13(1.2)$ \\
\hline East Timor & $1(0.1)$ & $3(0.3)$ \\
\hline Southern Asia & $60(7.5)$ & $130(12.3)$ \\
\hline India & $39(4.4)$ & $81(7.7)$ \\
\hline Bangladesh & $18(2.0)$ & $14(1.3)$ \\
\hline Maldives & $3(0.3)$ & $9(0.9)$ \\
\hline Sri Lanka & $7(0.8)$ & $26(2.5)$ \\
\hline Eastern Asia & $5(0.6)$ & $5(0.5)$ \\
\hline China & $3(0.3)$ & $1(0.1)$ \\
\hline Taiwan & $2(0.2)$ & $4(0.4)$ \\
\hline Others or unknown & $23(2.6)$ & $124(11.8)$ \\
\hline
\end{tabular}

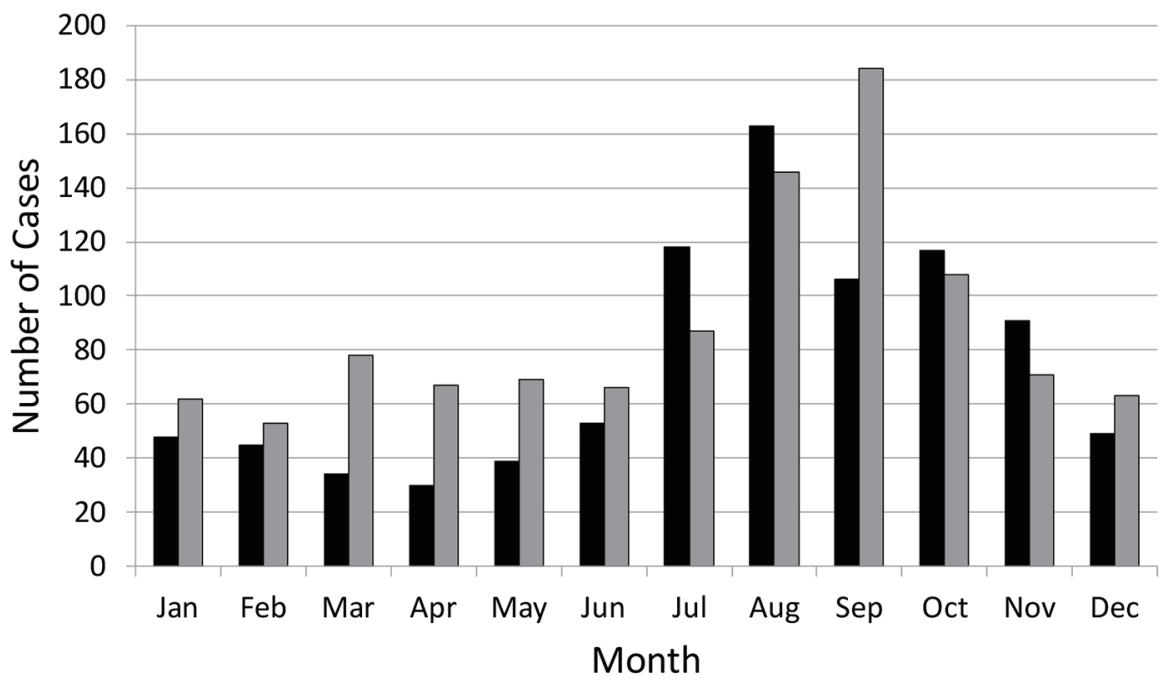

Figure 2. Distribution of imported dengue cases by month in Korea and Japan, 2011 - 2015. Bars represent the number of reported cases each month in Korea (black) and Japan (gray). 
than in developed countries [12-14]. Furthermore, dengue epidemics are not restricted to rural areas of such countries, as the habitat of Aedes aegypti has widened to urban residential areas, which travelers generally prefer visiting $[6,15]$.

As shown in Figure 2, the months with the most imported cases of dengue were in summer (June to August) and autumn (September to November) in both countries. The fact that the most active months for dengue infection in the Philippines are from July to October [14] is supposed to affect this result. Notably, August showed the highest number of cases in Korea, and September was highest in Japan. The slight difference implies differences in when people in each country commonly take holidays for travel and vacation. For example, university summer holidays in Korea are from July to August, while those in Japan are from August to September. These sorts of differences may result in different high-risk periods for mass dengue infection in the two countries.

Control systems for imported infectious diseases already exist in Korea and Japan, but the recent tendency toward greater worldwide travel increases the risk of dengue infection and, consequently, of imported dengue cases. Therefore, more rigorous information systems that effectively provide warning of the risk of dengue and means of prevention for travelers headed to at-risk regions are needed. We hope this study serves as a useful reference for developing more effective dengue control systems in both countries.

\section{Conflicts of Interest}

The authors declare no conflicts of interest relevant to this article.

\section{References}

1. World Health Organization (WHO). DENGUE, Guidelines for diagnosis, treatment, prevention and control. New edition, WHO, 2012. Available at $<$ https://www. ncbi.nlm.nih.gov/books/NBK143156/>. Accessed on December 19, 2016.

2. World Health Organization (WHO). Dengue and severe dengue. WHO, 2016. Available at <http://www.who.int/ mediacentre/factsheets/fs $117 / \mathrm{en} />$. Accessed on December 19, 2016.

3. Park JH, Lee DW. Dengue fever in South Korea, 20062010. Emerg Infect Dis. 2012;18(9):1525-1527.

4. Guzman MG, Harris E. Dengue. Lancet. 2015;385(9966):453-465.

5. Kim H, Lee C, Kim M. A case of imported dengue hemorrhagic fever. Kor J Infect Dis. 1995;27:403-406.

6. Takasaki T. Imported dengue fever/dengue hemorrhagic fever cases in Japan. Trop Med Health. 2011;39(4 Suppl):13-15.

7. Hotta S. Twenty years of laboratory experience with dengue virus. In: Saunders M, Lennette EH, eds. Medical and applied virology. St Louis: Green, 1965:228-256.

8. Kutsuna S, Kato Y, Moi ML, Kotaki A, Ota M, Shinohara $\mathrm{K}$, Kobayashi T, et al. Autochthonous dengue fever, Tokyo, Japan, 2014. Emerg Infect Dis. 2015;21(3):517-520.

9. Korea Center for Disease Control and Prevention (KCDC). Dengue fever. The Disease Web Statistical System. Available at $<$ https://is.cdc.go.kr/dstat/index.jsp $>$. Accessed on December 19, 2016.

10. National Institute of Infectious Diseases (NIID), Japan. Dengue Fever and dengue Hemorrhagic Fever: Notification trends among imported dengue cases in Japan, 20112015. Infectious Disease Surveillance Center (IDSC), NIID, Japan. Available at <http://www.nih.go.jp/niid/ja/ from-idsc.html $>$ Accessed on December 20, 2016.

11. Korean Statistical Information Service (KOSIS). Dengue fever. Available at $<\mathrm{http}$ ://kosis.kr/>. Accessed on December 19, 2016.

12. Korea Tourism Organization. Statistics of Korea Tourism. Available at <https://kto.visitkorea.or.kr/kor/notice/data/ statis.kto>. Accessed at December 19, 2016.

13. Japan Tourism Agency (JTA). Statistical information. Available at < http://www.mlit.go.jp/kankocho/siryou/ toukei/in_out.html $>$. Accessed on December 19, 2016.

14. Nakamura N, Arima Y, Shimada T, Matsui T, Tada Y, Okabe N. Incidence of dengue virus infection among Japanese travellers, 2006 to 2010. Western Pac Surveill Response J. 2012;3(2):39-45.

15. Murray NE, Quam MB, Wilder-Smith A. Epidemiology of dengue: past, present and future prospects. Clin Epidemiol. 2013;5:299-309. 